\title{
Weak-Strong Uniqueness for Compressible Magnetohydrodynamic Equations with Coulomb Force
}

\author{
Lianhua He $\mathbb{D}^{1,2}$ and Yonghui Zhou $\mathbb{D}^{3}$ \\ ${ }^{1}$ School of Mathematics Science, Guizhou Normal University, Guiyang 550001, China \\ ${ }^{2}$ School of Mathematical Sciences, Xiamen University, Xiamen 361005, China \\ ${ }^{3}$ School of big Data and Computer Science, Guizhou Normal University, Guiyang 550001, China
}

Correspondence should be addressed to Lianhua He; hlh1981@126.com

Received 22 March 2021; Accepted 20 June 2021; Published 28 July 2021

Academic Editor: Luigi C. Berselli

Copyright (c) 2021 Lianhua He and Yonghui Zhou. This is an open access article distributed under the Creative Commons Attribution License, which permits unrestricted use, distribution, and reproduction in any medium, provided the original work is properly cited.

In this paper, we consider the two-dimensional compressible magnetohydrodynamic system with Coulomb force. We apply the method of relative entropy to establish the weak-strong uniqueness property of this system.

\section{Introduction}

In this paper, we consider the compressible magnetohydrodynamic equations with Coulomb force as follows:

$$
\begin{gathered}
\partial_{t} \rho+\operatorname{div}(\rho u)=0 \\
\partial_{t}(\rho u)+\operatorname{div}(\rho u \otimes u)+\nabla\left(p+\frac{1}{2}|M|^{2}\right)-\mu \Delta u \\
-(\lambda+\mu) \nabla \operatorname{div} u=\operatorname{div}(M \otimes M)+\rho \nabla \Phi, \\
\partial_{t} M+\operatorname{div}(u \otimes M)-\operatorname{div}(M \otimes u)-v \Delta M=0, \operatorname{div} M=0,
\end{gathered}
$$

$$
\Delta \Phi=\rho-\bar{\rho} .
$$

Here $\rho, u, M$ and $\Phi$ stand for the electron density, electron velocity, magnetic field and electrostatic potential, respectively. $\bar{\rho}>0$ denotes the constant background ion density. $p=p(\rho)=a \rho^{\gamma}$ is the pressure function with the constant $a>0$ and the adiabatic exponent $\gamma>1$. The constants $\mu, \lambda$ are the viscosity coefficients satisfying $\mu>0$ and $\lambda+2 \mu / N \geq 0$ where $N$ is dimension. The constant $v>0$ is the magnetic diffusivity of the fluid. Let $\Omega \subset R^{2}$ be a bounded smooth domain. This system can be applied to describe the dynamics of a charge transport where the compressible electron fluid inter- acts with its own electric field against a charged ion background together under the influence of the magnetic field. Usually, we denominate the equation (1) as the continuity equation, the equation (2) as the momentum balance equation and the equation (3) as the induction equation. The motion of such fluids is driven by two dominating body forces, namely, the Coulomb force (i.e. the electric field force) and the Lorentz force imposed on the fluid by the magnetic field. This is the reason why the physical phenomena of such fluids is more complicated.

In this paper, we consider the initial-boundary conditions of MHD system (1)-(4) as following:

$$
\left\{\begin{array}{l}
\rho(0, x)=\rho_{0}(x) \in L^{\gamma}(\Omega), \rho_{0}(x) \geq 0 ; \int_{\Omega} \rho_{0}-\bar{\rho} d x=0 ; \\
\rho(0, x) u(0, x)=\left(\rho_{0} u_{0}\right)(x), \rho_{0}\left|u_{0}\right|^{2} \in L^{1}(\Omega) ; \\
M(0, x)=M_{0}(x) \in L^{2}(\Omega), \operatorname{div} M_{0}=0 \text { in } \mathscr{D}^{\prime}(\Omega) ; \\
\left.u\right|_{\partial \Omega}=0,\left.M\right|_{\partial \Omega}=0,\left.\nabla \Phi \cdot n\right|_{\partial \Omega}=0 .
\end{array}\right.
$$

where $n$ denotes the outer normal vector of $\partial \Omega$.

When there are no electromagnetic effect and no Coulomb force, the system (1)-(5) reduces to the compressible Navier-Stokes system. Many papers are devoted to the study 
of Navier-Stokes equations (see [1-5] and the references cited therein). These papers are focused on the existence of classical solution or weak solution and large time asymptotic behavior of solution et al. The weak-strong uniqueness is an important aspect in mathematical theory research of NavierStokes equations. The relative entropy method is an important method to research weak-strong uniqueness property of compressible Navier-Stokes equations. With the assistance of the concept of relative entropy, Germain [6] introduced a class of weak solution to the compressible Naiver-Stokes equations satisfying the relative entropy inequality with respect to a strong solution emanating from the same initial condition, and established the weak-strong uniqueness of this weak solution. Unfortunately, the existence of this class of weak solution is not known. Feireisl et al. [7] established the global existence of suitable weak solutions for any finite-energy initial data to compressible Navier-Stokes equations and proved the weak-strong uniqueness property within this class of suitable weak solutions by relative entropy inequality. By means of the relative entropy method, Feireisl et al. not only proved the weak-strong uniqueness of finiteenergy weak solution to compressible Navier-Stokes equations with monotone pressure in [8], but also proved the weak-strong uniqueness property for compressible NaiverStokes equations with non-monotone pressure in [9]. When the pressure function satisfies a hard sphere law, the weakstrong uniqueness of Navier-Stokes equations is established by Feireisl et al. in [10] and Chaudhur in [11] applying the method of relative entropy.

When there is no electromagnetic effect, the MHD system (1)-(5) reduces to the compressible Navier-StokesPoisson system. Many researchers are interested to study the compressible NSP system (see [12-17] and the references cited therein). These papers are focused on the existence and asymptotic behavior of solution et al. For the weeak-strong uniqueness of the compressible Navier-Stokes-Poisson system, we have discussed in [18].

Many researchers are interested in studying MHD system because of its physical importance, mathematical complexity and extensive applications(see [19-23] and references cited therein). There are also many references about the system (1)-(4)(see [19, 24-26]). In [24], Tan and Wang consider global existence and large time behavior of weak solution to the system (1)-(4). The existence of the finite-energy weak solution to the problem (1)-(5) is established by Feireisl et al. in [19] with $\gamma \geq 4 / 3$ and Jiang et al. in [25] with $(9+$ $\sqrt{33}) / 12<\gamma \leq 4 / 3$. Although the existence of weak solution has been established, the uniqueness problem is still an open problem. So far, there are very few results concerning the uniqueness of weak solutions to the initial-boundary value problem (1)-(5). Only when the dimension is three and the Coulomb force $\rho \nabla \Phi$ is not taken into account in the system (1)-(5), the weak-strong uniqueness of the finite-energy weak solution to the system (1)-(5) is established by Yang et al. in [26]. Then, our goal of this paper is to consider the uniqueness property of weak solution to two-dimensional MHD system with Coulomb force. In spirit of Feireisl [8], we will establish the uniqueness property of weak solution to the system (1)-(5) by using of the method of relative entropy. Compared with [8], the main difficulty is to deal with the items about magnetic field and electrostatic potential.

The paper is organized as follows. In Section 2, we recall the definition of finite-energy weak solution for the compressible MHD equations (1)-(5) and state the main results. In Section 3, we derive the relative entropy inequality to the system(1)-(5). In last section, we prove the weak-strong uniqueness property of the compressible MHD equations with Coulomb force (1)-(5).

\section{Main Results}

In this section, we define the finite-energy weak solution to the initial-boundary value problem (1)-(5) and state the main results.

Definition 1. $(\rho, u, \Phi, M)$ is called as a finite-energy weak solution of (1)-(5), if

(i) $(\rho, u, \Phi, M)$ belongs to the following class

$$
\begin{gathered}
\rho \geq 0, \rho \in L^{\infty}\left(0, T ; L^{\gamma}(\Omega)\right), \mathbf{u} \in L^{2}\left(0, T ; H_{0}^{1}(\Omega)\right), \\
\phi \in L^{\infty}\left(0, T ; H^{1}(\Omega)\right), \\
\mathbf{M} \in L^{\infty}\left(0, T ; L^{2}(\Omega)\right) \cap L^{2}\left(0, T ; H_{0}^{1}(\Omega)\right), \\
\quad \operatorname{div} \mathbf{M}(t)=0 \text { for } \text { a.a on } t \in(0, T) .
\end{gathered}
$$

(ii) $E(t) \in L_{l o c}^{1}(0, T)$ satisfies the energy inequality

$$
\frac{d}{d t} E(t)+\int_{\Omega}\left(\mu|\nabla u|^{2}+(\lambda+\mu)(\operatorname{div} u)^{2}+v|\nabla M|^{2}\right)(t, \cdot) d x \leq 0
$$

in $\mathfrak{D}^{\prime}(0, T)$, where the total energy $E(t)$ is defined as

$$
E(t)=\int_{\Omega}\left(\frac{1}{2} \rho|u|^{2}+\frac{a}{\gamma-1} \rho^{\gamma}+\frac{1}{2}|M|^{2}+\frac{1}{2}|\nabla \Phi|^{2}\right)(t, \cdot) d x .
$$

(iii) For any $t \in(0, T)$, the pair $(\rho, \Phi)$ satisfies the following Poisson equation

$$
\Delta \phi=\rho-\bar{\rho} \text { in } \Omega,\left.\nabla \phi \cdot \mathbf{n}\right|_{\partial \Omega}=0, \int_{\Omega} \phi d x=0 .
$$

(iv) Equation (1) holds in $\mathfrak{D}^{\prime}\left([0, T) \times R^{2}\right)$, i,e

$$
\begin{gathered}
\int_{\Omega} \rho(\tau, \cdot) \phi(\tau, \cdot) d x-\int_{\Omega} \rho_{0} \phi(0, \cdot) d x \\
=\int_{0}^{\tau} \int_{\Omega} \rho \partial_{t} \phi+\rho u \cdot \nabla \phi d x d t .
\end{gathered}
$$


for any $\phi \in C_{0}^{\infty}\left([0, T) \times R^{2}\right)$ and $\tau \in(0, T)$. Moreover, $(\rho, u)$ satisfies the renormalized equation

$$
\begin{aligned}
& \int_{\Omega}(b(\rho) \phi)(\tau, \cdot) d x-\int_{\Omega} b\left(\rho_{0}\right) \phi(0, \cdot) d x \\
&= \int_{0}^{\tau} \int_{\Omega} b(\rho) \partial_{t} \phi+b(\rho) u \cdot \nabla \phi \\
& \quad+\phi\left(b(\rho)-b^{\prime}(\rho) \rho\right) \operatorname{div} u d x d t .
\end{aligned}
$$

where $b \in C^{1}(R)$ such that

$$
b^{\prime}(z)=0 \text { for }|z| \text { large enough } .
$$

(v) Equation Equation (2) (3) holds in $\mathfrak{D}^{\prime}([0, T) \times \Omega)$, i,e

$$
\begin{aligned}
& \int_{\Omega} \rho u(\tau, \cdot) \cdot \varphi(\tau, \cdot) d x-\int_{\Omega} \rho_{0} u_{0} \cdot \varphi(0, \cdot) d x \\
& \quad+\int_{0}^{\tau} \int_{\Omega} \mu \nabla u: \nabla \varphi+(\lambda+\mu) \operatorname{div} u \operatorname{div} \varphi d x d t \\
& =\int_{0}^{\tau} \int_{\Omega} \rho u \cdot \partial_{t} \varphi+\rho u \otimes u: \nabla \varphi+p(\rho) \operatorname{div} \varphi \\
& \quad+\frac{1}{2}|M|^{2} \operatorname{div} \varphi-M \otimes M: \nabla \varphi+\rho \nabla \Phi \cdot \varphi d x d t . \\
& \quad \begin{array}{l}
M \\
\int_{\Omega}
\end{array} \\
& \quad+v(\tau, \cdot) d x-\int_{\Omega}^{\tau} \int_{\Omega} \nabla M: \varphi(0, \cdot) d x \\
& =\int_{0}^{\tau} \int_{\Omega} M \cdot \partial_{t} \varphi+u \otimes M: \nabla \varphi-M \otimes u: \nabla \varphi d x d t
\end{aligned}
$$

for any $\varphi \in C_{0}^{\infty}([0, T) \times \Omega)$.

Theorem 2. Assume that $\Omega \in R^{2}$ is a smooth domain. Let $\gamma$ $>9+\sqrt{33} / 12$. Then for any given $T>0$, there exist a finiteenergy weak solution $(\rho, u, \Phi, M)$ of the system (1)-(5).

\section{Remark 3.}

(1) When $\gamma>4 / 3$, we can apply the method in [19] without any modifications to prove the existence of finiteenergy weak solution

(2) When $9+\sqrt{33} / 12<\gamma \leq 4 / 3$, Jiang proved the existence of finite-energy weak solution $(\rho, u, \Phi, M)$ of the system (1)-(5) in [25].

Let $P(\rho)=(a / \gamma-1) \rho^{\gamma}$. Then the following equalities hold

$$
P^{\prime}(\rho) \rho-P(\rho)=p(\rho), P^{\prime \prime}(\rho) \rho=p^{\prime}(\rho) .
$$

Now we introduce main results of this paper.
Theorem 4. Let $(\rho, u, \Phi, M)$ be a finite-energy weak solution to the MHD system (1)-(5) in the sense of Definition 1. Let $r$, $\Psi \in C_{0}^{\infty}((0, T) \times \Omega)$ and $U, H \in C_{0}^{\infty}((0, T) \times \Omega)$. Assume that $(r, U, \Psi, H)$ satisfy

$$
\begin{gathered}
\partial_{t} H+\operatorname{div}(U \otimes H)-\operatorname{div}(H \otimes U)-v \Delta H=0, \operatorname{div} H=0 \\
\Delta \psi=r-\bar{\rho}, \partial_{t} r+\operatorname{div}(r \mathbf{U})=0,\left.\nabla \psi \cdot \mathbf{n}\right|_{\partial \Omega}=0
\end{gathered}
$$

Then the following relative entropy inequality holds for a.e $\tau \in(0, T)$

$$
\begin{aligned}
\varepsilon(\rho, u & , \Phi, M \mid r, U, \Psi, H)(\tau)+\int_{0}^{\tau} \int_{\Omega} \mu|\nabla(u-U)|^{2} \\
& +(\lambda+\mu)|\operatorname{div}(u-U)|^{2}+v|\nabla(M-H)|^{2} d x d t \\
\leq & \varepsilon\left(\rho_{0}, u_{0}, \Phi_{0}, M_{0} \mid r(0, \cdot), U(0, \cdot), \Psi(0, \cdot), H(0, \cdot)\right) \\
& +\int_{0}^{\tau} \mathscr{R}(t) d t
\end{aligned}
$$

where

$$
\begin{aligned}
& \varepsilon(\rho, u, \Phi, M \mid r, U, \Psi, H)(\tau) \\
&=\int_{\Omega}\left(\frac{1}{2} \rho|u-U|^{2}+P(\rho)-P^{\prime}(r)(\rho-r)-P(r)\right. \\
&\left.\quad+\frac{1}{2}|M-H|^{2}+\frac{1}{2}|\nabla(\Phi-\Psi)|^{2}\right)(\tau, \cdot) d x
\end{aligned}
$$

and the remainder $\mathscr{R}(t)$ is defined as

$$
\begin{aligned}
\mathscr{R}(t)= & \int_{\Omega} \mu \nabla U: \nabla(U-u)+(\lambda+\mu) \operatorname{div} U \operatorname{div}(U-u) d x \\
& +\int_{\Omega} \rho\left(\partial_{t} U+u \cdot \nabla U\right) \cdot(U-u) d x \\
& +\int_{\Omega}(H \otimes U-U \otimes H): \nabla(M-H) \\
& +(M \otimes u-u \otimes M): \nabla H+(U \otimes M-M \otimes U): \nabla M d x \\
& +\int_{\Omega} \rho(u-U) \cdot \nabla \Phi+(r U-\rho u) \cdot \nabla(\Phi-\Psi) d x \\
& +\int_{\Omega}(r-\rho) \partial_{t}\left(P^{\prime}(r)\right)+(r U-\rho u) \cdot \nabla\left(P^{\prime}(r)\right) \\
& +(p(r)-p(\rho)) \operatorname{div} U d x .
\end{aligned}
$$

Theorem 5. Let $\gamma>2$. Assume that $(\rho, u, \Phi, M)$ is the finiteenergy weak solution of the magnetohydrodynamic system (1)-(5) in the sense of Definition 1. And assume that $(r, U$, $\Psi, H)$ is the strong solution emanating from the same initial data and satisfy

$$
\begin{aligned}
& 0<\underline{r} \leq \inf _{(0, T) \times \Omega} r(t, x) \leq r(t, x) \leq \sup _{(0, T) \times \Omega} r(t, x)<\infty, \\
& U \in L^{2}\left(0, T ; W^{2, q}(\Omega)\right), \nabla^{2} U \in L^{1}\left(0, T ; L^{\infty}(\Omega)\right), \\
& H \in L^{4}\left(0, T ; W^{2, q}(\Omega)\right)
\end{aligned}
$$


with $q>2$. Then

$$
\rho=r, \mathbf{u}=\mathbf{U}, \phi=\psi, \mathbf{M}=\mathbf{H} \text { in }(0, T) \times \Omega .
$$

\section{Relative Entropy Inequality}

In this section, we will establish the relative entropy inequality(18) to the system (1)-(5) and extend the admissible class of test function.

\subsection{Proof of Theorem 4}

Proof. For simplicity, we define symbols $r_{0}, U_{0}, H_{0}$ as

$$
r_{0}=r(0, \cdot), \mathbf{U}_{0}=\mathbf{U}(0, \cdot), \mathbf{H}_{0}=\mathbf{H}(0, \cdot) .
$$

Taking $1 / 2|U|^{2}$ as test function in (10), we have

$$
\begin{aligned}
& \int_{\Omega} \frac{1}{2} \rho|U|^{2}(\tau, \cdot) d x-\int_{\Omega} \frac{1}{2} \rho_{0}\left|U_{0}\right|^{2} d x \\
& \quad=\int_{0}^{\tau} \int_{\Omega} \rho U \cdot \partial_{t} U+\rho u \cdot \nabla U \cdot U d x d t .
\end{aligned}
$$

Similarly, substituting $\varphi$ for $U$ as a test function in (13), we can get

$$
\begin{aligned}
\int_{\Omega}(\rho u \cdot U)(\tau, \cdot) d x-\int_{\Omega} \rho_{0} u_{0} \cdot U_{0} d x+\int_{0}^{\tau} \int_{\Omega} \mu \nabla u: \nabla U \\
\quad+(\lambda+\mu) \operatorname{div} u \operatorname{div} U d x d t \\
=\int_{0}^{\tau} \int_{\Omega} \rho u \cdot \partial_{t} U+\rho u \otimes u: \nabla U+p(\rho) \operatorname{div} U \\
\quad+\frac{1}{2}|M|^{2} \operatorname{div} U-M \otimes M: \nabla U+\rho \nabla \Phi \cdot U d x d t .
\end{aligned}
$$

Taking $\varphi=H$ in (14), we get

$$
\begin{aligned}
& \int_{\Omega}(M \cdot H)(\tau, \cdot) d x-\int_{\Omega} M_{0} \cdot H_{0} d x+v \int_{0}^{\tau} \int_{\Omega} \nabla M \\
& \quad: \nabla H d x d t=\int_{0}^{\tau} \int_{\Omega} M \cdot \partial_{t} H+u \otimes M: \nabla H-M \otimes u \\
& \quad: \nabla H d x d t .
\end{aligned}
$$

Multiplying (16) by $H$ and integrating over $(0, T) \times \Omega$, we can obtain

$$
\begin{gathered}
\int_{\Omega} \frac{1}{2}|H|^{2}(\tau, \cdot) d x-\int_{\Omega} \frac{1}{2}\left|H_{0}\right|^{2} d x+v \int_{0}^{\tau} \int_{\Omega}|\nabla H|^{2} d x d t \\
=\int_{0}^{\tau} \int_{\Omega} U \otimes H: \nabla H-H \otimes U: \nabla H d x d t .
\end{gathered}
$$

By virtue of $\Phi, \Psi$ satisfying (4) and (17), one has

$$
\Delta(\Phi-\Psi)=\rho-r .
$$

Differentiating (28) with respect to $t$, we get

$$
\partial_{t} \Delta(\Phi-\Psi)=\partial_{t} \rho-\partial_{t} r=\operatorname{div}(r U-\rho u) .
$$

Multiplying (29) by $(\Phi-\Psi)$ and integrating the resulting equation over $\Omega$, we can obtain

$$
\partial_{t}\left(\int_{\Omega} \frac{1}{2}|\nabla(\Phi-\Psi)|^{2} d x\right)=\int_{\Omega}(r U-\rho u) \cdot \nabla(\Phi-\Psi) d x .
$$

From (7), we can deduce

$$
\begin{aligned}
\int_{\Omega} & \left(\frac{1}{2} \rho|u|^{2}+\frac{a}{\gamma-1} \rho^{\gamma}+\frac{1}{2}|M|^{2}\right)(\tau, \cdot) d x \\
& \quad+\int_{0}^{\tau} \int_{\Omega} \mu|\nabla u|^{2}+(\lambda+\mu)|\operatorname{div} u|^{2}+v|\nabla M|^{2} d x d t \\
\leq & \int_{\Omega} \frac{1}{2} \rho_{0}\left|u_{0}\right|^{2}+\frac{a}{\gamma-1} \rho_{0}^{\gamma}+\frac{1}{2}\left|M_{0}\right|^{2} d x+\int_{0}^{\tau} \int_{\Omega} \rho u \cdot \nabla \Phi d x d t .
\end{aligned}
$$

Summing up relations (24)-(31), we can infer

$$
\begin{aligned}
\int_{\Omega}( & \left.\frac{1}{2} \rho|u-U|^{2}+P(\rho)+\frac{1}{2}|M-H|^{2}+\frac{1}{2}|\nabla(\Phi-\Psi)|^{2}\right)(\tau, \cdot) d x \\
& +\int_{0}^{\tau} \int_{\Omega} \mu \nabla u: \nabla(u-U)+(\lambda+\mu) \operatorname{div} u \operatorname{div}(u-U) d x d t \\
& +v \int_{0}^{\tau} \int_{\Omega}|\nabla M|^{2}-\nabla M: \nabla H+|\nabla H|^{2} d x d t \\
\leq & \int_{\Omega}\left(\frac{1}{2} \rho_{0}\left|u_{0}-U_{0}\right|^{2}+P\left(\rho_{0}\right)+\frac{1}{2}\left|M_{0}-H_{0}\right|^{2}\right. \\
& \left.+\frac{1}{2}\left|\nabla\left(\Phi\left(\rho_{0}\right)-\Psi\left(\rho_{0}\right)\right)\right|^{2}\right) d x \\
& +\int_{0}^{\tau} \int_{\Omega} \rho(U-u) \cdot \partial_{t} U+\rho u \cdot \nabla U \cdot(U-u)-p(\rho) \operatorname{div} U \\
& -\frac{1}{2}|M|^{2} \operatorname{div} U+M \otimes M: \nabla U d x d t \\
& -\int_{0}^{\tau} \int_{\Omega} M \cdot \partial_{t} H+u \otimes M: \nabla H-M \otimes u: \nabla H \\
& +H \otimes U: \nabla H-U \otimes H: \nabla H d x d t \\
& +\int_{0}^{\tau} \int_{\Omega} \rho(u-U) \cdot \nabla \Phi+(r U-\rho u) \cdot \nabla(\Phi-\Psi) d x d t .
\end{aligned}
$$

Note that

$$
\begin{aligned}
\int_{\Omega} & \left(-P^{\prime}(r)(\rho-r)-P(r)\right)(\tau, \cdot) d x \\
& -\int_{\Omega}\left(-P^{\prime}\left(r_{0}\right)\left(\rho_{0}-r_{0}\right)-P\left(r_{0}\right)\right) d x \\
= & \int_{0}^{\tau} \int_{\Omega} \partial_{t}\left(-P^{\prime}(r)(\rho-r)-P(r)\right) d x d t \\
= & \int_{0}^{\tau} \int_{\Omega}(r-\rho) \partial_{t}\left(P^{\prime}(r)\right)-\partial_{t} \rho P^{\prime}(r) d x d t \\
= & \int_{0}^{\tau} \int_{\Omega}(r-\rho) \partial_{t}\left(P^{\prime}(r)\right)-\rho u \cdot \nabla\left(P^{\prime}(r)\right) d x d t,
\end{aligned}
$$

$\int_{0}^{\tau} \int_{\Omega} r U \cdot \nabla\left(P^{\prime}(r)\right)+p(r) \operatorname{div} U d x d t=0$. 
Then we can rewrite (32) as

$$
\begin{aligned}
\int_{\Omega}( & \frac{1}{2} \rho|u-U|^{2}+P(\rho)-P^{\prime}(r)(\rho-r)-P(r)+\frac{1}{2}|M-H|^{2} \\
& \left.+\frac{1}{2}|\nabla(\Phi-\Psi)|^{2}\right)(\tau, \cdot) d x+\int_{0}^{\tau} \int_{\Omega} \mu \nabla u: \nabla(u-U) \\
& +(\lambda+\mu) \operatorname{div} u \operatorname{div}(u-U) d x d t \\
& +v \int_{0}^{\tau} \int_{\Omega}|\nabla M|^{2}-\nabla M: \nabla H+|\nabla H|^{2} d x d t \\
\leq & \int_{\Omega}\left(\frac{1}{2} \rho_{0}\left|u_{0}-U_{0}\right|^{2}+P\left(\rho_{0}\right)-P^{\prime}\left(r_{0}\right)\left(\rho_{0}-r_{0}\right)-P\left(r_{0}\right)\right. \\
& \left.+\frac{1}{2}\left|M_{0}-H_{0}\right|^{2}+\frac{1}{2}\left|\nabla\left(\Phi\left(\rho_{0}\right)-\Psi\left(\rho_{0}\right)\right)\right|^{2}\right) d x \\
& +\int_{0}^{\tau} \int_{\Omega} \rho\left(\partial_{t} U+u \cdot \nabla U\right) \cdot(U-u) d x d t \\
& +\int_{0}^{\tau} \int_{\Omega}^{\tau}-\frac{1}{2}|M|^{2} \operatorname{div} U+M \otimes M: \nabla U d x d t \\
& -\int_{0}^{\tau} \int_{\Omega} M \cdot \partial_{t} H+u \otimes M: \nabla H-M \otimes u: \nabla H \\
& +H \otimes U: \nabla H-U \otimes H: \nabla H d x d t \\
& +\int_{0}^{\tau} \int_{\Omega}^{\tau} \rho(u-U) \cdot \nabla \Phi+(r U-\rho u) \cdot \nabla(\Phi-\Psi) d x d t \\
& +\int_{0}^{\tau} \int_{\Omega}(r-\rho) \partial_{t}\left(P^{\prime}(r)\right)+(r U-\rho u) \cdot \nabla\left(P^{\prime}(r)\right) \\
& -(p(\rho)-p(r)) \operatorname{div} U d x d t .
\end{aligned}
$$

For simplicity, we abbreviate $\varepsilon(\rho, u, \Phi, M \mid r, U, \Psi, H)(\tau)$ to $\varepsilon(\tau)$.

Replacing $\partial_{t} H$ with (16), we can infer

$$
\begin{aligned}
\varepsilon(\tau)+ & \int_{0}^{\tau} \int_{\Omega} \mu \nabla u: \nabla(u-U)+(\lambda+\mu) \operatorname{div} u \operatorname{div}(u-U) \\
& +v|\nabla(M-H)|^{2} d x d t \\
\leq & \varepsilon(0)+\int_{0}^{\tau} \int_{\Omega} \rho\left(\partial_{t} U+u \cdot \nabla U\right) \cdot(U-u) d x d t \\
& +\int_{0}^{\tau} \int_{\Omega}(H \otimes U-U \otimes H): \nabla M+(M \otimes u-u \otimes M): \nabla H \\
& +(U \otimes H-H \otimes U): \nabla H d x d t+\int_{0}^{\tau} \int_{\Omega} \rho(u-U) \cdot \nabla \Phi \\
& +(r U-\rho u) \cdot \nabla(\Phi-\Psi) d x d t+\int_{0}^{\tau} \int_{\Omega}(r-\rho) \partial_{t}\left(P^{\prime}(r)\right) \\
& +(r U-\rho u) \cdot \nabla\left(P^{\prime}(r)\right)-(p(\rho)-p(r)) \operatorname{div} U d x d t \\
& +\int_{0}^{\tau} \int_{\Omega} U \otimes M: \nabla M-M \otimes U: \nabla M d x d t .
\end{aligned}
$$

Finally, we can get

$$
\begin{aligned}
\varepsilon(\tau)+ & \int_{0}^{\tau} \int_{\Omega} \mu|\nabla(u-U)|^{2}+(\lambda+\mu)|\operatorname{div}(u-U)|^{2} \\
& +\nu|\nabla(M-H)|^{2} d x d t \leq \varepsilon(0)+\int_{0}^{\tau} \mathscr{R}(t) d t .
\end{aligned}
$$

The proof of Theorem 4 is completed.

3.2. Extending the Admissible Class of Test Function. Making use of density argument, we can extend the class of test function $(r, U, \Psi, H)$ appeared in the relative entropy inequality (18), (20).

For the left hand side of (18) to be well defined, the function $(r, U, \Psi, H)$ must belong at least to the class:

$$
\begin{gathered}
r \in C\left([0, T] ; L_{\omega}^{\gamma}(\Omega)\right), \mathbf{U} \in C\left([0, T] ; L_{\omega}^{2 \gamma / \gamma-1}(\Omega)\right) ; \\
\nabla \mathbf{U} L^{2}((0, T) \times \Omega),\left.\mathbf{U}\right|_{\partial \Omega}=0 ; \\
\mathbf{H} \in L^{2}\left(0, T ; H_{0}^{1}(\Omega)\right) \cap C\left([0, T] ; L_{\omega}^{2}(\Omega)\right) ; \\
\Psi \in C\left([0, T] ; W_{\omega}^{2, \gamma}(\Omega)\right),\left.\nabla \Psi \cdot n\right|_{\partial \Omega}=0 .
\end{gathered}
$$

Similarly, a short inspection of the integrals in (20) yields

$$
\begin{gathered}
\partial_{t} \mathbf{U} \in L^{1}\left(0, T ; L^{2 \gamma / \gamma-1}(\Omega)\right) ; \nabla \mathbf{U} \in L^{1}\left(0, T ; L^{\infty}(\Omega)\right) ; \\
\nabla \mathbf{H} \in L^{2}\left((0, T) ; L^{\infty}(\Omega)\right) .
\end{gathered}
$$

Moreover, the function $r$ must be bounded away from zero, and

$\partial_{t}\left(P^{\prime}(r)\right) \in L^{1}\left(0, T ; L^{\gamma / \gamma-1}(\Omega)\right) ; \nabla\left(P^{\prime}(r)\right) \in L^{1}\left(0, T ; L^{2 \gamma / \gamma-1}(\Omega)\right)$.

It is easy to prove that the relative entropy inequality (18), (20) can be extended to $(r, U, \Psi, H)$ satisfying (37)-(42) by density argument.

\section{Weak-Strong Uniqueness}

In this section, we will prove weak-strong uniqueness property of the MHD system (1)-(5). In order to prove the weak-strong uniqueness, we firstly rewrite the remainder $\mathscr{R}(t)$ as another expression. Then we apply Gronwall's inequality to prove the weak-strong uniqueness property.

\subsection{Another Expression of $\mathscr{R}(t)$}

Lemma 6. Let $(r, U, \Phi, H)$ be a strong solution to MHD system (1)-(5). For $r \geq \underline{r}>0$, the remainder $\mathscr{R}(t)$ can be rewritten as 


$$
\begin{aligned}
\mathscr{R}(t)= & \int_{\Omega} \rho(u-U) \cdot \nabla U \cdot(U-u) d x \\
& -\int_{\Omega}\left(p(\rho)-p^{\prime}(r)(\rho-r)-p(r)\right) \operatorname{div} U d x \\
& +\int_{\Omega}(u-U) \cdot \nabla H \cdot(H-M)+(M-H) \cdot \nabla H \\
& \cdot(u-U) d x+\int_{\Omega}(M-H) \cdot \nabla(H-M) \cdot U+U \\
& \cdot \nabla(M-H) \cdot(M-H) d x+\int_{\Omega} \frac{\rho-r}{r}(U-u) \\
& \cdot(\mu \Delta U+(\lambda+\mu) \nabla \operatorname{div} U) d x+\int_{\Omega} \frac{\rho-r}{r}(H \cdot \nabla H \\
& \cdot(U-u)-(U-u) \cdot \nabla H \cdot H) d x \\
& +\int_{\Omega}(r-\rho) U \cdot \nabla(\Phi-\Psi) d x .
\end{aligned}
$$

Proof. Because $(r, U, \Phi, H)$ is a strong solution to MHD system (1)-(5), we can get

$$
\begin{aligned}
\partial_{t} U+U \cdot \nabla U= & r^{-1}(\mu \Delta U+(\lambda+\mu) \nabla \operatorname{div} U)-r^{-1} \nabla(p(r)) \\
& -r^{-1} \nabla\left(\frac{|H|^{2}}{2}\right)+r^{-1} \operatorname{div}(H \otimes H)+\nabla \Psi
\end{aligned}
$$

From (44), the remainder $\mathscr{R}(t)$ is rewritten as

$$
\begin{aligned}
\mathscr{R}(t)= & \int_{\Omega} \frac{\rho-r}{r}(U-u) \cdot(\mu \Delta U+(\lambda+\mu) \nabla \operatorname{div} U) d x \\
& +\int_{\Omega} \frac{\rho}{r}(U-u) \cdot\left(-\nabla\left(\frac{|H|^{2}}{2}\right)+\operatorname{div}(H \otimes H)\right) d x \\
& +\int_{\Omega} \rho(u-U) \cdot \nabla U \cdot(U-u) d x \\
& +\int_{\Omega}(H \otimes U-U \otimes H): \nabla(M-H) \\
& +(M \otimes u-u \otimes M): \nabla H+(U \otimes M-M \otimes U): \nabla M d x \\
& +\int_{\Omega} \rho(u-U) \cdot \nabla(\Phi-\Psi)+(r U-\rho u) \cdot \nabla(\Phi-\Psi) d x \\
& +\int_{\Omega}(r-\rho) \partial_{t}\left(P^{\prime}(r)\right)+(r-\rho) U \cdot \nabla\left(P^{\prime}(r)\right) d x \\
& +\int_{\Omega}(p(r)-p(\rho)) \operatorname{div} U d x .
\end{aligned}
$$

Note that

$(r-\rho) \partial_{t}\left(P^{\prime}(r)\right)+(r-\rho) U \cdot \nabla\left(P^{\prime}(r)\right)=p^{\prime}(r)(\rho-r) \operatorname{div} U$
Then we can rewrite $\mathscr{R}(t)$ as

$$
\begin{aligned}
\mathscr{R}(t)= & \int_{\Omega} \frac{\rho-r}{r}(U-u) \cdot(\mu \Delta U+(\lambda+\mu) \nabla \operatorname{div} U) d x \\
& +\int_{\Omega} \frac{\rho}{r}(U-u) \cdot\left(-\nabla\left(\frac{|H|^{2}}{2}\right)+\operatorname{div}(H \otimes H)\right) d x \\
& +\int_{\Omega} \rho(u-U) \cdot \nabla U \cdot(U-u) d x \\
& +\int_{\Omega}(H \otimes U-U \otimes H): \nabla(M-H) \\
& +(M \otimes u-u \otimes M): \nabla H+(U \otimes M-M \otimes U): \nabla M d x \\
& +\int_{\Omega}(r-\rho) U \cdot \nabla(\Phi-\Psi) d x \\
& -\int_{\Omega}\left(p(\rho)-p^{\prime}(r)(\rho-r)-p(r)\right) \operatorname{div} U d x .
\end{aligned}
$$

Besides, it is easy to infer that

$$
\begin{aligned}
\int_{\Omega}- & \frac{\rho}{r}(U-u) \cdot \nabla\left(\frac{|H|^{2}}{2}\right)+\frac{\rho}{r}(U-u) \cdot \operatorname{div}(H \otimes H) d x \\
= & \int_{\Omega} \frac{\rho-r}{r}(H \cdot \nabla H \cdot(U-u)-(U-u) \cdot \nabla H \cdot H) d x \\
& +\int_{\Omega} H \cdot \nabla H \cdot(U-u)-(U-u) \cdot \nabla H \cdot H d x
\end{aligned}
$$

$$
\begin{aligned}
\int_{\Omega}(H \otimes U-U \otimes H): \nabla(M-H)+(M \otimes u-u \otimes M): \nabla H \\
\quad+(U \otimes M-M \otimes U): \nabla M d x \\
\quad+\int_{\Omega} H \cdot \nabla H \cdot(U-u)-(U-u) \cdot \nabla H \cdot H d x \\
=\int_{\Omega} u \cdot \nabla H \cdot(H-M)+(M-H) \cdot \nabla H \cdot u \\
\quad+U \cdot \nabla M \cdot(M-H)+(H-M) \cdot \nabla M \cdot U d x \\
=\int_{\Omega}(u-U) \cdot \nabla H \cdot(H-M)+U \cdot \nabla H \cdot(H-M) \\
\quad+U \cdot \nabla M \cdot(M-H) d x \\
\quad+\int_{\Omega}(M-H) \cdot \nabla H \cdot(u-U) \\
\quad+(M-H) \cdot \nabla H \cdot U+(H-M) \cdot \nabla M \cdot U d x \\
=\int_{\Omega}(u-U) \cdot \nabla H \cdot(H-M)+U \cdot \nabla(M-H) \\
\quad \cdot(M-H) d x+\int_{\Omega}(M-H) \cdot \nabla(H-M) \\
\quad \cdot U+(M-H) \cdot \nabla H \cdot(u-U) d x .
\end{aligned}
$$


On the basis of (48) and (49), we can infer

$$
\begin{aligned}
\mathscr{R}(t)= & \int_{\Omega} \rho(u-U) \cdot \nabla U \cdot(U-u) d x \\
& -\int_{\Omega}\left(p(\rho)-p^{\prime}(r)(\rho-r)-p(r)\right) \operatorname{div} U d x \\
& +\int_{\Omega}(u-U) \cdot \nabla H \cdot(H-M)+(M-H) \cdot \nabla H \\
& \cdot(u-U) d x+\int_{\Omega} U \cdot \nabla(M-H) \cdot(M-H) \\
& +(M-H) \cdot \nabla(H-M) \cdot U d x+\int_{\Omega} \frac{\rho-r}{r}(U-u) \\
& \cdot(\mu \Delta U+(\lambda+\mu) \nabla \operatorname{div} U) d x+\int_{\Omega} \frac{\rho-r}{r} \\
& \cdot(H \cdot \nabla H \cdot(U-u)-(U-u) \cdot \nabla H \cdot H) d x \\
& +\int_{\Omega}(r-\rho) U \cdot \nabla(\Phi-\Psi) d x .
\end{aligned}
$$

The proof of Lemma 6 is completed.

4.2. Proof of Theorem 5. To begin, it is easy to check that

$P(\rho)-P^{\prime}(r)(\rho-r)-P(r) \geq c(r) \begin{cases}(\rho-r)^{2}, & \text { for } \frac{r}{2} \leq \rho \leq 2 r \\ \left(1+\rho^{\gamma}\right), & \text { otherwise. }\end{cases}$

where $c(r)$ is uniformly bounded for $r$ belonging to compact sets in $(0, \infty)$.

Proof. There are two steps to prove Theorem 5.

Step 1: We estimate the remainder $\mathscr{R}(t)$.

For $\nabla U \in L^{1}\left(0, T ; L^{\infty}(\Omega)\right)$ and $(51)$, it is easy to infer that

$$
\begin{aligned}
& \quad \mid \int_{\Omega} \rho(u-U) \cdot \nabla U \cdot(U-u) d x \\
& \quad-\int_{\Omega}\left(p(\rho)-p^{\prime}(r)(\rho-r)-p(r)\right) \operatorname{div} U d x \mid \\
& \quad \leq C\|\nabla U\|_{L^{\infty}(\Omega)} \varepsilon(t) \leq \eta(t) \varepsilon(t) .
\end{aligned}
$$

By using of Hölder's inequality and Sobolev's inequality, we can deduce

$$
\begin{aligned}
& \left|\int_{\Omega}(\mathbf{u}-\mathbf{U}) \cdot \nabla \mathbf{H} \cdot(\mathbf{H}-\mathbf{M}) d x+\int_{\Omega}(\mathbf{M}-\mathbf{H}) \cdot \nabla \mathbf{H} \cdot(\mathbf{u}-\mathbf{U}) d x\right| \\
& \leq C \int_{\Omega}|\mathbf{u}-\mathbf{U}\|\nabla \mathbf{H}\|(\mathbf{M}-\mathbf{H})| d x \leq C\|\mathbf{M}-\mathbf{H}\|_{L^{2}(\Omega)}\|\nabla \mathbf{H}\|_{L^{q}(\Omega)} \\
& \quad \cdot\|\mathbf{u}-\mathbf{U}\|_{L^{2 q / q-2}(\Omega)} \leq C\|\mathbf{M}-\mathbf{H}\|_{L^{2}(\Omega)}\|\nabla \mathbf{H}\|_{L^{q}(\Omega)} \\
& \quad \cdot\|\mathbf{u}-\mathbf{U}\|_{w_{0}^{1,2}(\Omega)} \leq C\|\nabla \mathbf{H}\|_{L^{q}(\Omega)}^{2} \int_{\Omega}|\mathbf{M}-\mathbf{H}|^{2} d x \\
& \quad+\delta \int_{\Omega}|\nabla(\mathbf{u}-\mathbf{U})|^{2} d x \leq \eta(t) \varepsilon(t)+\delta \int_{\Omega}|\nabla(\mathbf{u}-\mathbf{U})|^{2} d x
\end{aligned}
$$

$$
\begin{aligned}
& \left|\int_{\Omega} U \cdot \nabla(M-H) \cdot(M-H) d x\right| \\
& \quad=\left|-\int_{\Omega} \frac{|M-H|^{2}}{2} \operatorname{div} U d x\right| \\
& \quad \leq C\|\operatorname{div} U\|_{L^{\infty}(\Omega)} \varepsilon(t) \leq \eta(t) \varepsilon(t)
\end{aligned}
$$

here $\delta>0$ is sufficient small and $q>2$.

For $\operatorname{div} M=\operatorname{div} H=0$, it is easy to get that

$$
\begin{aligned}
& \left|\int_{\Omega}(M-H) \cdot \nabla(H-M) \cdot U d x\right|=\mid-\int_{\Omega}(M-H) \cdot \nabla U \\
& \quad \cdot(H-M) d x\left|\leq C\|\nabla U\|_{L^{\infty}(\Omega)} \int_{\Omega}\right| M-\left.H\right|^{2} d x \leq \eta(t) \varepsilon(t) .
\end{aligned}
$$

Now, we estimate the last three items of $\mathscr{R}(t)$.

$$
\begin{aligned}
I= & \int_{\Omega} \frac{\rho-r}{r}(\mu \Delta U+(\lambda+\mu) \nabla \operatorname{div} U)(U-u) d x \\
= & \left(\int_{\{r / 2 \leq \rho \leq 2 r\}}+\int_{\{0 \leq \rho<r / 2\}}+\int_{\{\rho>2 r\}}\right) \frac{\rho-r}{r} \\
& \cdot(\mu \Delta U+(\lambda+\mu) \nabla \operatorname{div} U)(U-u) d x:=I_{1}+I_{2}+I_{3} .
\end{aligned}
$$

We can make use of Hölder's inequality, Sobolev's inequality and (51) to obtain

$$
\begin{aligned}
\left|I_{1}\right| & =\left|\int_{\{r / 2 \leq \rho \leq 2 r\}} r^{-1}(\rho-r)(\mu \Delta U+(\lambda+\mu) \nabla \operatorname{div} U)(U-u) d x\right| \\
& \leq C\left\|\nabla^{2} U\right\|_{L^{q}(\Omega)}\|\rho-r\|_{L^{2}(\{r / 2 \leq \rho \leq 2 r\})}\|U-u\|_{L^{2 q / q-2}(\Omega)} \\
& \leq C\left\|\nabla^{2} U\right\|_{L^{q}(\Omega)}\|\rho-r\|_{L^{2}(\{r / 2 \leq \rho \leq 2 r\})}\|U-u\|_{W_{0}^{1,2}(\Omega)} \\
& \leq C\left\|\nabla^{2} U\right\|_{L^{q}(\Omega)}^{2} \int_{\{r / 2 \leq \rho \leq 2 r\}}(\rho-r)^{2} d x+\delta \int_{\Omega}|\nabla(U-u)|^{2} d x \\
& \leq \eta(t) \varepsilon(t)+\delta \int_{\Omega}|\nabla(U-u)|^{2} d x,
\end{aligned}
$$

$$
\begin{aligned}
\left|I_{2}\right|= & \left|\int_{\{0 \leq \rho<r / 2\}} r^{-1}(\rho-r)(U-u)(\mu \Delta U+(\lambda+\mu) \nabla \operatorname{div} U) d x\right| \\
\leq & C\left\|\nabla^{2} U\right\|_{L^{q}(\Omega)}\|1\|_{L^{2}(\{0 \leq \rho<r / 2\})}\|U-u\|_{L^{2 q / q-2}(\Omega)} \\
\leq & C\left\|\nabla^{2} U\right\|_{L^{q}(\Omega)}\|1\|_{L^{2}(\{0 \leq \rho<r / 2\})}\|U-u\|_{W_{0}^{1,2}(\Omega)} \\
\leq & C\left\|\nabla^{2} U\right\|_{L^{q}(\Omega)}^{2} \int_{\{0 \leq \rho<r / 2\}} P(\rho)-P^{\prime}(r)(\rho-r)-P(r) d x \\
& +\delta \int_{\Omega}|\nabla(U-u)|^{2} d x \leq \eta(t) \varepsilon(t)+\delta \int_{\Omega}|\nabla(U-u)|^{2} d x .
\end{aligned}
$$

where $q>2$. 
Note that

$$
\begin{aligned}
\left\|\rho^{\gamma / 2}\right\|_{L^{2}(\{\rho>2 r\})} & \leq(\varepsilon(t))^{1 / 2} \text { and }\left|\frac{\rho-r}{\rho r}\right| \rho^{1 / 2-\gamma / 2} \\
& \leq C \text { as } \rho>2 r \geq 2 \underline{r}>0, \gamma>1 .
\end{aligned}
$$

By Hölder's inequality and (57), we can obtain

$$
\begin{aligned}
\left|I_{3}\right|= & \left|\int_{\{\rho>2 r\}} \frac{\rho-r}{r}(U-u)(\mu \Delta U+(\lambda+\mu) \nabla \operatorname{div} U) d x\right| \\
\leq & C \int_{\{\rho>2 r\}}\left|\frac{\rho-r}{\rho r} \rho^{1 / 2-\gamma / 2}\right| \cdot \rho^{\gamma / 2} \cdot|\mu \Delta U+(\lambda+\mu) \nabla \operatorname{div} U| \\
& \cdot \rho^{1 / 2}|U-u| d x \\
\leq & C\left\|\nabla^{2} U\right\|_{L^{\infty}(\Omega)}\left(\int_{\{\rho>2 r\}} \rho^{\gamma} d x\right)^{1 / 2}\left(\int_{\Omega} \rho|U-u|^{2} d x\right)^{1 / 2} \\
\leq & \eta(t) \varepsilon(t) .
\end{aligned}
$$

Therefor, we can get the estimate of $I$

$$
|I| \leq \eta(t) \varepsilon(t)+\delta \int_{\Omega}|\nabla(U-u)|^{2} d x
$$

with $\delta>0$ sufficiently small.

Similarly to the estimate of $I$, we write

$$
\begin{aligned}
J= & \int_{\Omega} \frac{\rho-r}{r}(H \cdot \nabla H \cdot(U-u)-(U-u) \cdot \nabla H \cdot H) d x \\
= & \left(\int_{\{r / 2 \leq \rho \leq 2 r\}}+\int_{\{0 \leq \rho<r / 2\}}+\int_{\{\rho>2 r\}}\right) \frac{\rho-r}{r} \\
& \cdot(H \cdot \nabla H \cdot(U-u)-(U-u) \cdot \nabla H \cdot H) d x:=J_{1}+J_{2}+J_{3} .
\end{aligned}
$$

Applying Hölder's inequality, Sobolev's inequality, (51) and (57), we can infer that

$$
\begin{aligned}
\left|J_{1}\right| & =\left|\int_{\{r / 2 \leq \rho \leq 2 r\}} \frac{\rho-r}{r}(H \cdot \nabla H \cdot(U-u)-(U-u) \cdot \nabla H \cdot H) d x\right| \\
& \leq \int_{\left\{\frac{r}{2} \leq \rho \leq 2 r\right\}}|\rho-r| \cdot|H| \cdot|\nabla H| \cdot|U-u| d x \\
& \leq C\|H\|_{L^{\infty}(\Omega)}\|\nabla H\|_{L^{q}(\Omega)}\|\rho-r\|_{L^{2}(\{r / 2 \leq \rho \leq 2 r\})}\|U-u\|_{L^{2 q / q-2}(\Omega)} \\
& \leq C\|H\|_{W^{1, q}(\Omega)}^{2}\|\rho-r\|_{L^{2}(\{r / 2 \leq \rho \leq 2 r\})}\|U-u\|_{W_{0}^{1,2}(\Omega)} \\
& \leq C\|H\|_{W^{1, q}(\Omega)}^{4} \int_{\{r / 2 \leq \rho \leq 2 r\}}|\rho-r|^{2} d x+\delta \int_{\Omega}|\nabla(U-u)|^{2} d x \\
& \leq \eta(t) \varepsilon(t)+\delta \int_{\Omega}|\nabla(U-u)|^{2} d x
\end{aligned}
$$

$$
\begin{aligned}
\left|J_{2}\right| & =\left|\int_{\{0 \leq \rho<r / 2\}} \frac{\rho-r}{r}(H \cdot \nabla H \cdot(U-u)-(U-u) \cdot \nabla H \cdot H) d x\right| \\
& \leq \int_{\{0 \leq \rho<r / 2\}} 1 \cdot|H| \cdot|\nabla H| \cdot|U-u| d x \\
& \leq C\|H\|_{L^{\infty}(\Omega)}\|\nabla H\|_{L^{q}(\Omega)}\|1\|_{L^{2}(\{0 \leq \rho<r / 2\})}\|U-u\|_{L^{2 q / q-2}(\Omega)} \\
& \leq C\|H\|_{W^{1, q}(\Omega)}\|1\|_{L^{2}(\{0 \leq \rho<r / 2\})}\|U-u\|_{W_{0}^{1,2}(\Omega)} \\
& \leq C\|H\|_{W^{1, q}(\Omega)}^{4} \int_{\{0 \leq \rho<r / 2\}} 1 d x+\delta \int_{\Omega}|\nabla(U-u)|^{2} d x \\
& \leq \eta(t) \varepsilon(t)+\delta \int_{\Omega}|\nabla(U-u)|^{2} d x ; \\
\left|J_{3}\right| & =\left|\int_{\{\rho>2 r\}} \frac{\rho-r}{r}(H \cdot \nabla H \cdot(U-u)-(U-u) \cdot \nabla H \cdot H) d x\right| \\
& \leq C \int_{\{\rho>2 r\}}\left|\frac{\rho-r}{\rho r} \rho^{1 / 2-\gamma / 2}\right| \cdot \rho^{\gamma / 2} \cdot|H| \cdot|\nabla H| \cdot \rho^{1 / 2}|U-u| d x \\
& \leq C\|H\|_{L^{\infty}(\Omega)}\|\nabla H\|_{L^{\infty}(\Omega)}\left\|\rho^{\gamma / 2}\right\|_{L_{(\{\rho>2 r\})}^{2}}\left\|\rho^{1 / 2}(U-u)\right\|_{L^{2}(\Omega)} \\
& \leq C\|H\|_{W^{2, q}(\Omega)}^{4} \varepsilon(t) \leq \eta(t) \varepsilon(t) .
\end{aligned}
$$

with $q>2$.

Finally, we estimate the last item of $\mathscr{R}(t)$.

$$
\begin{aligned}
K= & \int_{\Omega}(r-\rho) U \cdot \nabla(\Phi-\psi) d x \\
= & \left(\int_{\{r / 2 \leq \rho \leq 2 r\}}+\int_{\{0 \leq \rho<r / 2\}}+\int_{\{\rho>2 r\}}\right)(r-\rho) U \\
& \cdot \nabla(\Phi-\psi) d x:=K_{1}+K_{2}+K_{3} .
\end{aligned}
$$

From (51), we can get

$$
\begin{aligned}
\left|K_{1}\right| \leq & \int_{\{r / 2 \leq \rho \leq 2 r\}}|r-\rho||\mathrm{U}| \cdot|\nabla(\Phi-\psi)| d x \\
\leq & C\|U\|_{L^{\infty}(\Omega)}\|r-\rho\|_{L^{2}(\{r / 2 \leq \rho \leq 2 r\})}\|\nabla(\Phi-\psi)\| \\
\leq & C\|U\|_{L^{\infty}(\Omega)}\left(\int_{r / 2 \leq \rho \leq 2 r} P(\rho)-P^{\prime}(r)(\rho-r)-P(r) d x\right)^{1 / 2} \\
& \cdot(\varepsilon(t))^{1 / 2} \leq \eta(t) \varepsilon(t) ; \\
\left|K_{2}\right| \leq & \int_{\{0 \leq \rho<r\}} 1 \cdot|U| \cdot|\nabla(\Phi-\psi)| d x \\
\leq & C\|U\|_{L^{\infty}(\Omega)}\|1\|_{L^{2}(\{0 \leq \rho<r\})}\|\nabla(\Phi-\psi)\| \\
\leq & C\|U\|_{L^{\infty}(\Omega)}\left(\int_{0 \leq \rho<r} P(\rho)-P^{\prime}(r)(\rho-r)-P(r) d x\right)^{1 / 2} \\
& \cdot(\varepsilon(t))^{1 / 2} \leq \eta(t) \varepsilon(t) .
\end{aligned}
$$


Using of Hölder's inequality and (57), we can infer that

$$
\begin{aligned}
\left|K_{3}\right| & =\left|\int_{\{\rho>2 r\}}(r-\rho) U \cdot \nabla(\Phi-\psi) d x\right| \\
& \leq C \int_{\{\rho>2 r\}}\left|\rho^{\gamma / 2}\right| \cdot|U| \cdot|\nabla(\Phi-\psi)| d x \\
& \leq C\|U\|_{L^{\infty}(\Omega)}\left\|\rho^{\gamma / 2}\right\|_{L^{2}(\{\rho>2 r\})}\|\nabla(\Phi-\psi)\| \leq \eta(t) \varepsilon(t)
\end{aligned}
$$

with $\gamma>2$.

Step 2: Making use of Gronwall's inequality, we can prove the weak-strong uniqueness of MHD system (1)-(5).

Substituting inequalities (52)-(64) into (18), we can obtain

$$
\begin{aligned}
\varepsilon(\tau) & +\int_{0}^{\tau} \int_{\Omega} \mu|\nabla(u-U)|^{2}+(\lambda+\mu)|\operatorname{div}(u-U)|^{2} \\
& +v|\nabla(M-H)|^{2} d x d t \leq \int_{0}^{\tau} \eta(t) \varepsilon(t) d t
\end{aligned}
$$

with $\eta(t) \in L^{1}(0, T)$. By Gronwall's inequality, we can deduce $\varepsilon(t)=0$ in $(0, T)$. Then this implies $\rho=r, u=U, \Phi=\Psi, M=$ $H$. The proof of Theorem 5 is completed.

Remark 7. The strong solution we work with in the weakstrong uniqueness theorem is only a hypothetical object which we do not know if it even exist. In the future, we will consider the existence of this strong solution.

\section{Data Availability}

The data that support the findings of this study are available from the corresponding author upon reasonable request.

\section{Conflicts of Interest}

The authors declare that they have no conflicts of interest.

\section{Acknowledgments}

The work of He and Zhou was supported by China NSF grant (11861025).

\section{References}

[1] E. Feireisl, Dynamicals of Viscous Compressible Fluids, Oxford University Press, Oxford, UK, 2004.

[2] E. Feireisl, A. Novotný, and H. Petzeltová, “On the existence of globally defined weak solutions to the Navier-Stokes equations," Journal of Mathematical Fluid Mechanics, vol. 3, no. 4, pp. 358-392, 2001.

[3] J. Li and Z. P. Xin, "Global well-posedness and large time asymptotic behavior of classical solutions to the compressible Navier-Stokes equations with vacuum," Annals of PDE, vol. 5, no. 1, 2019.

[4] P. L. Lions, Mathematical Topic in Fluid Dynamics, Vol 2, Compressible Models, Oxford Science Publication, Oxford, UK, 1998.
[5] A. Novotný and I. Straskraba, Introduction to the theory of compressible flow, Oxford University Press, Oxford, UK, 2004.

[6] P. Germain, "Weak-strong uniqueness for the isentropic compressible Navier-Stokes system," Journal of Mathematical Fluid Mechanics, vol. 13, no. 1, pp. 137-146, 2011.

[7] E. Feireisl, A. Novotný, and Y. Sun, "Suitable weak solutions to the Navier-stokes equations of compressible viscous fluids," Indiana University Mathematics Journal, vol. 60, no. 2, pp. 611-632, 2011.

[8] E. Feireisl, B. Jin, and A. Novotný, "Relative Entropies, suitable weak solutions, and weak-strong uniqueness for the compressible Navier-stokes system," Journal of Mathematical Fluid Mechanics, vol. 14, no. 4, pp. 717-730, 2012.

[9] E. Feireisl, "On weak-strong uniqueness for the compressible Navier-Stokes system with non-monotone pressure law," Communications in Partial Differential Equations, vol. 44, no. 3, pp. 271-278, 2019.

[10] E. Feireisl, Y. Lu, and A. Novotný, "Weak-strong uniqueness for the compressible Navier-Stokes equations with a hardsphere pressure law," Science China Mathematics, vol. 61, no. 11, pp. 2003-2016, 2018.

[11] N. Chaudhuri, "On Weak-strong uniqueness for compressible Navier-Stokes system with general pressure laws," Nonlinear Analysis: Real World Applications, vol. 49, pp. 250-267, 2019.

[12] Q. Bie, Q. Wang, and Z. Yao, "Optimal decay rate for the compressible Navier-Stokes-Poisson system in the critical $L^{P}$ framework," Journal of Differential Equations, vol. 263, no. 12, pp. 8391-8417, 2017.

[13] C. Hao and H. L. Li, "Global existence for compressible Navier-stokes-Poisson equations in three and higher dimensions," Journal of Differential Equations, vol. 246, no. 12, pp. 4791-4812, 2009.

[14] T. Kobayashi and T. Suzuki, "Weak solution to the Navierstokes-Poisson equation," Advances in Mathematical Sciences and Applications, vol. 18, pp. 141-168, 2008.

[15] H. L. Li, A. Matsumura, and G. Zhang, "Optimal decay rate of the compressible Navier-stokes-Poisson system in $\mathbb{R}^{3}$," Archive for Rational Mechanics and Analysis, vol. 196, no. 2, pp. 681-713, 2010.

[16] Y. J. Wang, "Decay of the Navier-stokes-Poisson equations," Journal of Differential Equations, vol. 253, no. 1, pp. 273297, 2012.

[17] Y. H. Zhang and Z. Tan, "On the existence of solutions to the Navier-stokes-Poisson equations of a two-dimensional compressible flow," Mathematical Methods in the Applied Sciences, vol. 30, no. 3, pp. 305-329, 2007.

[18] L. H. He and Z. Tan, "Weak-strong uniqueness for the NavierStokes-Poisson equations," Applied Mathematics Letters, vol. 103, article 106143, 2020.

[19] B. Ducomet and E. Feireisl, "The equations of Magnetohydrodynamics: on the interaction between matter and radiation in the evolution of gaseous stars," Communications in Mathematical Physics, vol. 266, no. 3, pp. 595-629, 2006.

[20] J. Fan and W. Yu, "Global variational solutions to the compressible magnetohydrodynamic equations," Nonlinear Analysis: Theory, Methods \& Applications, vol. 69, no. 10, pp. 3637-3660, 2008.

[21] X. Hu and D. Wang, "Global solutions to the threedimensional full compressible magnetohydrodynamic flows," Communications in mathematical physics, vol. 283, no. 1, pp. 255-284, 2008. 
[22] B. Lü, Z. H. Xu, and X. Zhong, "Global existence and large time asymptotic behavior of strong solutions to the Cauchy problem of 2D density-dependent magnetohydrodynamic equations with vacuum," Journal de Mathématiques Pures et Appliquées, vol. 108, no. 1, pp. 41-62, 2017.

[23] M. Yu, "Global classical solutions to the 2D compressible MHD equations with large data and vacuum," Journal of Differential Equations, vol. 258, no. 9, pp. 3304-3359, 2015.

[24] Z. Tan and Y. J. Wang, "Global existence and large-time behavior of weak solutions to the compressible magnetohydrodynamic equations with coulomb force," Nonlinear Analysis, vol. 71, no. 11, pp. 5866-5884, 2009.

[25] F. Jiang, Z. Tan, and H. Q. Wang, "A note on global existence of weak solutions to the compressible magnetohydrodynamic equations with coulomb force," Journal of Mathematical Analysis and Applications, vol. 379, no. 1, pp. 316-324, 2011.

[26] Y. F. Yang, C. Dou, and Q. Ju, "Weak-strong uniqueness property for the magnetohydrodynamic equations of threedimensional compressible isentropic flows," Nonlinear Analysis, vol. 85, pp. 23-30, 2013. 